\title{
Transforming research: engaging patient advocates at all stages of cancer research
}

\author{
AnneMarie Ciccarella ${ }^{1}$, Alicia C. Staley ${ }^{2}$, Aime T. Franco ${ }^{3}$ \\ ${ }^{1}$ Independent Patient Research Partner, New York, NY, USA; ${ }^{2}$ Akari Health, Charlestown, MA, USA; ${ }^{3}$ Department of Physiology and Biophysics, \\ University of Arkansas for Medical Sciences, Little Rock, AR, USA \\ Correspondence to: Aime T. Franco. Department of Physiology and Biophysics, Biomedical Research Bld. II Room 264-2, Little Rock, AR 72205, USA. \\ Email: ATFranco@uams.edu.
}

\begin{abstract}
In recent years there has been a push to include patients and advocates in clinical research, including in clinical trial design and approval processes. Additionally, increased advocate-driven research funding has led to seats for advocates in the funding review process. However, despite advances that include having patients and advocates involved in clinical research projects that may directly, or eventually, affect their care and treatment, we have much further to go to make this a truly inclusionary participation in research. We have even further to go to actively incorporate patients and advocates throughout the research continuum. Here we review and present current programs and projects that have successfully incorporated patients and advocates into translational research, and propose mechanism and strategies to actively engage patients throughout the research continuum. Our goal in translational cancer research is to improve outcomes for cancer patients. To more successfully and quickly meet this objective we should incorporate patient driven research questions earlier in the process, specifically at the level of basic benchtop research.
\end{abstract}

Keywords: Cancer; advocacy; patient centered-outcomes

Submitted Apr 25, 2018. Accepted for publication Apr 26, 2018.

doi: $10.21037 /$ atm.2018.04.46

View this article at: http://dx.doi.org/10.21037/atm.2018.04.46

Scientists seek to solve problems. In cancer, the most important problems are those of the patients. Often the patient perspective and insight can give investigators a new lens to look at a problem. Patient participation in research has grown, but primarily in the clinical research setting. There is a growing understanding within the clinical realm, that patients, the stakeholders in their own care, should be involved with studies that impact their health. Engagement is beginning to be implemented at multiple stages of the research spectrum, from experimental design through review of grants to fund these studies and dissemination of the results. Unfortunately, the pace is slow to actively include patients throughout the spectrum of clinical cancer research, and patients are often viewed to be passive participants to "check boxes" of patient relevance or patient-centered approaches. The pace is even slower in engaging patients at earlier stages in the research continuum, especially at the preclinical and basic research level. Little has been written about the relationship between patients and research, particularly in basic research, and the need for engaging patients in translational research early in the research continuum. Here we present both the researcher and patients' prospective on avenues and strategies to actively engage patients early in the research continuum and discuss methods by which patients are rewriting the protocols on research design and development of basic science programs, for patients by patients.

Many grant reviews, especially for clinical or late stage translational studies, include a patient advocate on the panel of reviewers. However, their role is often viewed as one to validate adequate protections are in place for patients involved in research. This is a lost opportunity. Instead of merely commenting on safety and justification of risks, the patients and advocates should be actively participating and commenting on the relevance and potential outcomes of the study to the patients. Are the questions being asked relevant 
to the patient? Are there other concerns or outcomes that would interest a patient? In our goal of trying to discern as much information from trials and understand both the mechanisms that drive successful treatment and mechanisms of resistance or failure of therapy, we need to be cognizant of the risks to and commitment of the patient. We must balance the knowledge gained, with the demands that we place upon the patient for participation, including increased monitoring and time to contribute to the study. Survival is not the only end point important for patients; quality of life and maintaining as normal a life as possible are critical.

We must seek input from patients to determine which interventions, but more importantly, which procedures and protocols are feasible for a patient, and balance answering the scientific research questions with maintaining reasonable expectations of patient participation. As scientists we seek as much data from a study as we can obtain, but this can be at the expense of considering how this data collection affects the patient. A recent commentary in Nature points to the need for incorporation of "robust evaluation for outcomes that truly matter to patients" (1). The best way to achieve this goal is through partnerships with patient advocates who can speak for the populations we seek to help when conducting our research. Repeated biopsies and scans to track therapeutic response are vital to collecting data to understand mechanisms of action and resistance, but we must not lose sight of pain and consequences to the patient of repeated clinic visits, biopsies and scans. Adequate protections are not enough. Patients must be actively involved in study and trial design to provide insight as to reasonable expectations. We are dependent upon patients to be willing to participate in clinical trials, and we must be aware and sensitive to the sacrifices that patients make to participate in research. By empowering patients to be partners throughout the research continuum, we may be able to start answering questions at much earlier phases of research, particularly in well-controlled environment of the research laboratory, and thus be able to better predict outcomes within the clinic and design more patient-centric trials.

We need to avoid having patients and advocates being viewed as token requirements, and lead by including them as an active part of the research team that provide unique perspectives and fulfill roles that most scientists and researchers cannot. Unfortunately, inclusion of patients and advocates is often not a scored criterion in many research proposals. The mission of the NIH is "to seek fundamental knowledge about the nature and behavior of living systems and the application of that knowledge to enhance health, lengthen life, and reduce illness and disability" (2). There needs to be a balance on what knowledge we seek, while actively seeking to involve the stakeholders whose lives will be the ultimate beneficiaries of that research. This will not be an easy fix. We must change the way we see collaborations and our research teams. We need to encourage and facilitate students and postdocs to interact with patients and advocates so that they view these individuals as equal stakeholders, and recognize the value that they contribute to our research programs at all stages of investigation. Patients have already been involved in a number of research related activities and have in some instances, co-authored papers on decision making tools (3).

In discussions with many patients whose advocacy efforts are research focused, they are attempting to incorporate what they learn from their participation at scientific meetings or from serving on review panels. They believe the proper time to engage is when we are in the process of developing the research questions within the lab. While patients are quick to acknowledge they are not scientists, they share their frustration about the specificity of the questions being asked in the laboratory. What if the questions we are asking are not aligned with the needs of the patient populations we seek to help? It would be best to have a deeper understanding of what might be most helpful for those patients and populations early in experimental design to attempt to see these needs are met whenever possible. Inclusion/exclusion criteria are another area where the input of patients would be most valuable. Designing trials with a third arm in ways that would afford otherwise ineligible patients to participate would afford us the opportunity to glean additional insights that could benefit both patients and researchers. Data analysis and results would not be skewed by this additional population arm if it was appropriately incorporated into the study design from the onset of the trial. Patients can and should play a role in analysis and interpretation of results. They are also the most valuable resource that a researcher has for dissemination of study results to the communities that will be impacted by the research.

With the increasing incorporation of patient reported outcomes into trial design, the patient voice is essential in helping design pertinent questions. As we see a focus on "real-world evidence," patient advocate partners are likely better equipped to bring this information to researchers and clinicians to help guide the next questions that need to be answered. Too many patients may hesitate to share 
some of the effects of their treatment, sometimes out of fear, others not sure of the relevance of their concerns. In some cases, they don't want to be seen as a difficult patient, or burdening their providers with what may be critical information. Unfortunately, more frequently, there is an underlying fear that patients will be forced to stop a treatment that seems to be working. Clear communication and transparency is critical to any relationship: patientphysician; patient-researcher; physician-researcher; and this communication and collaboration must be established early in the research continuum so that all the concerns and priorities of each participant can be met. Collaboration between well-informed advocates, communities they serve and the medical community would help mitigate this communication gap.

Partnerships between researchers and advocates should be expanded into all phases of our studies. These partnerships should not be limited to only those studies whose funding mechanism requires the inclusion of an advocate. Likewise, the skills of a qualified advocate provide value in even the most basic science or in research involving the development or the incorporation of highly technical diagnostic and prognostic tools. Although basic science, including successful observations we might see on slides or in animal models occurs many years before first in human trials, including advocates in bench experiments is invaluable. Most highly effective advocates may not fully grasp the details behind the science they seek to advance and are quick to acknowledge their greatest strength lays in their ability to "be smart enough to know, and admit what they don't know." Without getting lost in those scientific details upon which researchers must keep in sharp focus, advocates possess the unique ability to remain focused on the impact and significance for patients. As we develop, for example, assays or animal models requiring the donation of blood or other biospecimens for basic science, our patient partners have the ability to see what the successful translation of these studies might mean to affected populations, even if the benefits may not occur until years later. They are focused on the bigger picture which includes the possibility of less invasive testing, more accurate assessment of disease burden, the identification of targets that would afford patients the knowledge to help guide treatment decisions at various points in time while minimizing or eliminating the need for repeated invasive testing. As we continue to study molecular aberrations, protein interactions, the tumor microenvironment and many other areas, our research advocate partnerships help shed light on how the most basic science discoveries and highly technological advances hold promise toward enhancing the quality of life from routine diagnostic screening to diagnostic and prognostic screening, through active treatment and for the remainder of their lives. We learn from patient partnerships that they would like a crystal ball. They are plagued with questions that can only be answered by conducting basic research: Is the treatment working? What are the potential late effects of the treatment? Has the cancer recurred or metastasized? What options are available to afford the best outcomes for longevity while simultaneously preserving quality of life? Patient concerns and research objectives can only be aligned when all voices are brought to the table as a concept is brought from discussion to the development and design stage, and throughout the course of experimentation. Frequent engagement to discuss both the achievement of aims and where we experience pitfalls helps keep the research nimble and focused on the ultimate goal: the delivery of the promise of precision medicine for the best personalized care available.

The key to any successful collaborative efforts is to establish the appropriate team, and to define the role of each member. This is critical to patient/advocate-scientist relationships, and to recognize the expertise that each partner brings to the project. A common misconception that has emerged among scientists is that patients and advocates will try to design or dictate experiments. This is not the role of the patient in this relationship. Instead, the patient brings with them the knowledge of the questions that are important to the patient. Often these are questions that a basic scientist would not seek to answer, or had not even been aware was an unmet or unanswered need within the field. Each cancer and survivor story is different, and depending on type of disease, prognosis and availability of current therapies, patient research priorities are very different. A prime example of this came while participating in the Dr. Susan Love Research Foundation Metastatic Breast Cancer Collateral Damage Think Tank. The discussion turned to searching for a cure for metastatic breast cancer. We naively thought that a cure is what was needed and what patients desired. However, the patients made clear that a cure was not the only option, nor necessarily the desired outcome: that they wanted more options, more treatments available so that when they ran out of options with the currently available drugs, they had something that could get them to the next milestone: a birthday, an anniversary, perhaps even another clinical breakthrough. This insight completely changed the way that we now ask questions in 
the laboratory, and also changes the way that we interpret our results and data. Successful treatment as measured by absolute resolution of the tumor is no longer the "gold standard" but improvement over what is currently available, or disruption of a new pathway/unique pathway to those targeted with the current standards of care is now viewed as a success. This may seem like an obvious endpoint to those practicing clinical measurements or involved with clinical trials, but rarely are our metrics for success or endpoints of success in laboratory preclinical models aligned with clinical endpoints of trials. This discrepancy can drive the lack of concordance between preclinical and clinical investigations. If we realigned our metrics and endpoints to be more common, then the challenges of moving from preclinical to clinical would be lessened. We must ask patients and learn about the questions that are important to them, so that we can change our preclinical and basic science research design and metrics. Why do we wait to address patient centric questions until clinical practice when we can begin to address these needs and questions much earlier in the spectrum at basic science investigations? This was simultaneously a haunting and enlightening experience, as it became glaringly obvious how misaligned research goals can be with patient goals and outcomes.

Research is grueling. Many experiments don't work. The rewards of meeting patients and putting a human perspective to the research work that we do can be life changing. Knowing the futures that you are making brighter because of the hours spent at the bench is amazing. Further, the human component supports how important all discoveries are, including the discovery of approaches that do not work. It is critical for scientists and patients to understand the dual struggles that we face: researchers need to publish and submit their work and results for peer review, while patients seek as much information as they can, and seek hope that there are new therapies and approaches on the horizon. This provides an ideal intersection for collaboration. Patients and advocates know the need for new therapies and knowledge, and also understand the need and value to report negative outcomes so that we do not needlessly slow progress and repeat trials and experiments that have marginal effect. Patients demand the need for reporting all clinical trial data. They can and should demand the same for all research, and highlight that even studies and research that do not support improved outcomes is important to drive research forward, and prevent needless repetition of experiments that will not positively impact patient care.
Central to the development of collaborative relationships and projects between scientists and advocates, is to share common knowledge and language. Unfortunately, researchers are often not directly involved with clinical care of the diseases that they investigate, nor do they always have direct personal experience with that disease. Patients and advocates, although well versed in their own personal condition, do not always have a broader knowledge of cancer biology, nor have an understanding of the research process. These initial barriers must be overcome to establish meaningful, collaborative relationships. Researchers often have the opportunity to interact with clinicians directly treating the disease that they investigate, and to participate in Grand Rounds and clinical seminar series to understand the clinical management of their disease of interest. However, the greater challenge is for patients and advocates to seek educational opportunities to learn more about cancer biology and the research process. Fortunately, many national societies, and patient advocacy groups have recognized the need for educational opportunities for advocates, and programs have been developed.

Researchers need to assist with helping educate patients and advocates so that they can be informed and educated participants within the research community. Most patients do not come from a science or medical background, and therefore need additional information and education so that they can more actively participate in the research process in an informed manner. This is an ideal opportunity to involve early career scientists and trainees to help develop programs and educational tools that can be utilized by cancer patients and advocates to learn the basics of cancer biology, and to ensure that all research participants are using the same language. The public, patients and advocacy groups can be invited to Graduate Student Research Days. This gives students and scientists the opportunity to describe their work and discoveries, and allows patients and community members the opportunity to see the exciting work taking place locally at research institutions.

Some national organizations have built and developed formal programs to educate patients and advocates. For 20 years the American Association for Cancer Research (AACR) has been engaging advocates with scientists at their Annual Meeting (and now expanded to Special Meetings and Workshops) through the Scientist $\leftrightarrow$ Survivor Program (SSP) (4). Advocates are assigned to small groups and paired with both an advocate mentor and a scientist mentor. Scientific mentors place the research findings presented at the meeting into context of where this research falls into the 
research continuum, and to provide insight as to how basic discoveries made in the laboratory can be translated into clinical practice. It is critical for patients and advocates to see firsthand the work that is being done by scientists across the world to improve care and therapeutic options for cancer patients, but to also understand the research process, and the time and effort expended long before a new drug ever is introduced into a clinical trial. The SSP program allows many patients and advocates their first opportunity to ever discuss research with a bench scientist. But more importantly, having advocates and patients at the AACR Annual Meeting, allows scientists; particularly graduate students and postdocs the opportunity to interact with patients and advocates that are impacted by the diseases that they research in the laboratory, and often for the first times puts a human face to the potential of who their research may impact. Participation in the SSP program led to establishment of the Franco Lab "Why we are here?" board. This board is at the entrance to the laboratory and serves as a daily reminder that all that is done in the laboratory is for the benefit of the patient, and is driven by the patient. Our research may not change clinical practice tomorrow, but the results and the questions that we ask in the laboratory are all driven by the desire to improve outcomes for cancer patients. It is critical to recognize that improved outcomes are not always about life and death, but about quality of life and improving the treatment options that are available. The board also serves as a sober reminder that despite the many "thrivers" recognized on the board, we also have lost many friends and family members because our current therapies failed them, and that we must strive for better.

The United States' Department of Defense Congressionally Directed Medical Research Program has incorporated patients or caregivers on their review panels. The U.S. National Cancer Institute (NCI) also includes patient advocates on their review panels. The establishment of the U.S. Office of Advocate Relations (OAR) to properly vet and identify interested advocates and pair them with researchers. A gap exists in these programs. While advocates are invited to serve in the review process, only some funding mechanisms require their participation in the research. Patient Centered Outcomes Research Institute (PCORI) has shown the strongest level of support by incorporating advocates on all levels, encouraging researchers to identify and name qualified advocates as coinvestigators on their funding submissions.

Many patient advocacy groups have also seen the need to help educate advocates into the scientific process. Some are disease specific, such as Project LEAD from the
National Breast Cancer Coalition (5), the CADRE program through The Prostate Net (6), and Alamo Breast Cancer Foundation runs a scholarship program in conjunction with the San Antonio Breast Cancer Symposium. However, we need to develop strategies and programs so that we can educate and target more advocates, especially at the local level so that they can become involved with research at their local institutions. Attendance of meetings is expensive and often not accessible to all advocates and patients. We need programs to bring the access of these programs into the community, and increase the population of informed advocates that can actively participate in research.

Programs have also been developed to support advocates in their work, and to provide continuing education in scientific areas. BAG IT is a grassroots project begun in Tucson, Arizona, USA by survivors, for survivors (7). BAG IT provides cancer patients, regardless of age, gender or cancer type with information at the time of diagnosis to assist them with navigating the early days of cancer diagnosis, and how to empower themselves to be more involved with their care and advocating for their own personal best outcomes. BAG IT also recognized the need to further educate and care for advocates involved in their own program and mission, as well as cancer advocates at large assisting patients and cancer survivors. This led to the development of ESCAPE to Thrive, a program for advocates to promote collaboration, education and self-care. This retreat for advocates provides educational programing about current and upcoming policies and practices affecting oncology, cancer care and survivorship, new scientific and research advances, and practices for improved well-being, both physically and psychologically.

Patients and advocates are able to often look at questions and research problems through a lens, not jaded by years of failed experiments or tedious protocols, but instead through a lens of optimism and hope for better outcomes, and for a brighter future with new options. This optimism and alternative lens is desperately needed in the laboratory, especially during times of fiscal crisis, when so much great research is left unfunded. We must strive to find a balance between funding great science, and funding discovery and questions that are risky, and have the most potential to directly impact the patients that we are ultimately trying to help. The best way to ensure we are asking questions relevant to patients is to ask the patients right from the start! It takes a village, and meaningful participation and input from all that reside in that village to drive change and progress. 


\section{Acknowledgements}

We wish to thank and dedicate this manuscript to the many patients and advocates who have selflessly contributed to scientific discovery through their participation in research. In particular, Aime Franco would like to thank Lori MarxRubiner for her friendship and insight. She had the insight to challenge this scientist/cancer survivor to see research through a different lens, and to embrace the lens of being a patient-scientist.

\section{Footnote}

Conflicts of Interest: The authors have no conflicts of interest to declare.

\section{References}

1. Aggarwal A. Demand cancer drugs that truly help patients.

Cite this article as: Ciccarella A, Staley AC, Franco AT. Transforming research: engaging patient advocates at all stages of cancer research. Ann Transl Med 2018;6(9):167. doi: 10.21037/ atm.2018.04.46
Nature 2018;556:151.

2. NIH. NIH Mission, 2018. Available online: https://www. nih.gov/about-nih/what-we-do/mission-goals

3. Attai DJ, Cowher MS, Al-Hamadani M, et al. Twitter social media is an effective tool for breast cancer patient education and support: patient-reported outcomes by survey. J Med Internet Res 2015;17:e188.

4. Research AACR. AACR Scientist Survivor Program, 2018. Available online: http:// www.aacr.org/ADVOCACYPOLICY/ SURVIVORPATIENTADVOCACY/PAGES/ SCIENTISTHARR\%3BSURVIVORPROGRAM_403E94.ASPX\#.Wto9UC7waM8

5. Project LEAD, 2018. Available online: http://www. breastcancerdeadline2020.org/get-involved/training/ project-lead/

6. CADRE, 2018. Available online: http://www.prostateonline.com/knowledge.html.

7. Bag It. 2018. Available online: https://bagit4u.org/ 